\title{
Self-organizing Analysis on the Urban Agglomeration System Based on Synergy Innovation
}

\author{
Ai-jun LIU ${ }^{1,2, a}$ and Shu-juan $\mathrm{LI}^{3, \mathrm{~b},{ }^{*}}$ \\ ${ }^{1}$ School of Business, Wuhan Huaxia University of Technology, China \\ ${ }^{2}$ School of Management, Hubei University of Education, China \\ ${ }^{3}$ School of Economics and Management, Wuhan Sports University, China \\ a65871825@qq.com, bshujuanziru@163.com
}

Keywords: Urban agglomeration, Synergy innovation, Self-organization.

\begin{abstract}
The competition of urban agglomeration is the main form of regional economic development; synergy innovation is an effective form of urban agglomeration development. The self-organization mechanism of synergy innovation system of urban agglomeration was analyzed; the synergy model of innovation subjects was established based on the Synergy to demonstrate the motivation and inevitability of the synergy innovation. From the perspective of the orderly evolution and the control evolution, the self-organization process of the synergy innovation of urban agglomeration was discussed. The Order parameter is the deciding factor, the control parameter affects the process and results, and they complete the self-organization under the interaction.
\end{abstract}

\section{Introduction}

Innovation is the main driving force of the social economic development, regional innovation system is the extension and embodiment of the national innovation system in the regional level. British economist Philip Cooke proposed regional innovation system in the 1990s [1]. Veroniea Serrano (2007) believes that the key point of regional synergy innovation is that the system innovation elements are highly synergy and flow and diffusion [2]. Shen Yuming (1999) thinks that urban agglomeration can be integrated into the world economy only through competition and cooperation under the background of economic integration [3]. Xie Xuemei (2011) analyzed the city circle of collaborative innovation process, innovation system in the role of the order parameter is crucial, it will affect the development direction of system self-organization [4]. Ren Jianhua believed that the synergy between subsystems could promote the system to produce a better stable solution and the growth rate of the subsystem [5]. Cheng Qiang thought that the self-organization of subsystems made the system form super circulation [6].The research on synergy innovation has been more detailed both here and abroad, but the self-organization process of urban agglomeration system is rarely discussed from the macro-perspective and the micro-analysis.

\section{The Main Element of Synergy Innovation of the Urban Agglomeration}

The main element of synergy innovation of the urban agglomeration includes the government, the enterprise, the university and research institution, the service organization. The government as one of the main elements does not directly participate in synergy innovation activities, but its role can't be replaced. In the initial stage, the government plays a leading role due to its special function of guidance and coordination. 
The government formulates, regulates, improves and implements the corresponding policy system, organizes and regulates the rational allocation of resources, the coordination and protection of the interests of the subject of innovation and other issues. Because of its obvious profit, the enterprise is the largest source of power for the entire innovation activities, research and develop new products, technologies and services, which continuously feedbacks and transforms the technology innovation achievements into products or services to achieve profits, thus promoting economic and social development. The university plays an indispensable role because of its outstanding human and scientific research resources, and its main functions including the talents cultivation and the technology development. The research institution mainly engages in applied research and basic research oriented application, it is one of the knowledge sources of technology innovation, but also the main contributors to the knowledge base, can provide scientific and technological achievements directly to enterprises. Due to this similar function of the $R \& D$, the research institution and the university cooperate and penetrated mutually cooperation, which is not uncommon. The service organization services, communicates, notarizes and supervises, directly involves in the technology innovation of service object or provides advisory and resources allocation services.

In addition, functional elements including the technology, the resource, the talent and the capital, environmental elements such as the system, the law and the culture are also important parts of the urban agglomeration system and will affect the synergy innovation system.

\section{Synergy Analysis on the Urban Agglomeration Innovation}

\section{The Synergy Feature of the Innovation Subject of the Urban Agglomeration}

The research object is regarded as a combination of a large number of subsystems, which interact with each other through the exchange of material, energy and information. The innovation system of urban agglomeration is such a synergy system. Urban agglomeration is composed of nodes (enterprises, scientific research institutions, universities, etc.), links between nodes (network, link), flows (talent, material, information, capital). It depends on the interaction of different innovation subjects in the innovation system. The nonlinear interaction among the elements in the innovation system is the internal power and source of the self-organization evolution, synergy is the form and means of self-organization.

Self-development. The unstable principle of the Synergetics points out that the formation of a new model means the instability of the original model. Urban agglomeration system is rooted in scarcity of structural resources of the city and the complementarity and symbiosis of elements. Different innovation subject integrate materials, knowledge, information, technology, products, talents and exchange to make the system far from the equilibrium. As a result, the old structure is constantly removed and a new structure is established.

Self-adaptation. The innovation system of the urban agglomeration shows the autonomy, self-stabilizing and self-coordination facing external inputs. It automatically absorbs energy, accelerates the elements integration, to produce synergistic effect and form the synergy mechanism of element-subject-environment, achieve the survival of the fittest and Orderly evolution. 
Self-evolution. When the system constantly adapt to the external environment, the system's stochastic "fluctuation" force at the critical point of the system will be a small fluctuations magnified into a macrofluctuation

When the system continually adapts to the external environment, random "fluctuation" power of the system will enlarge a tiny fluctuation to become huge fluctuation at a critical point, which is shown in Fig.1.Expansion and fluctuation is a basic effect, which could be produced by technological changes, product innovation, institutional and environment evolution etc.

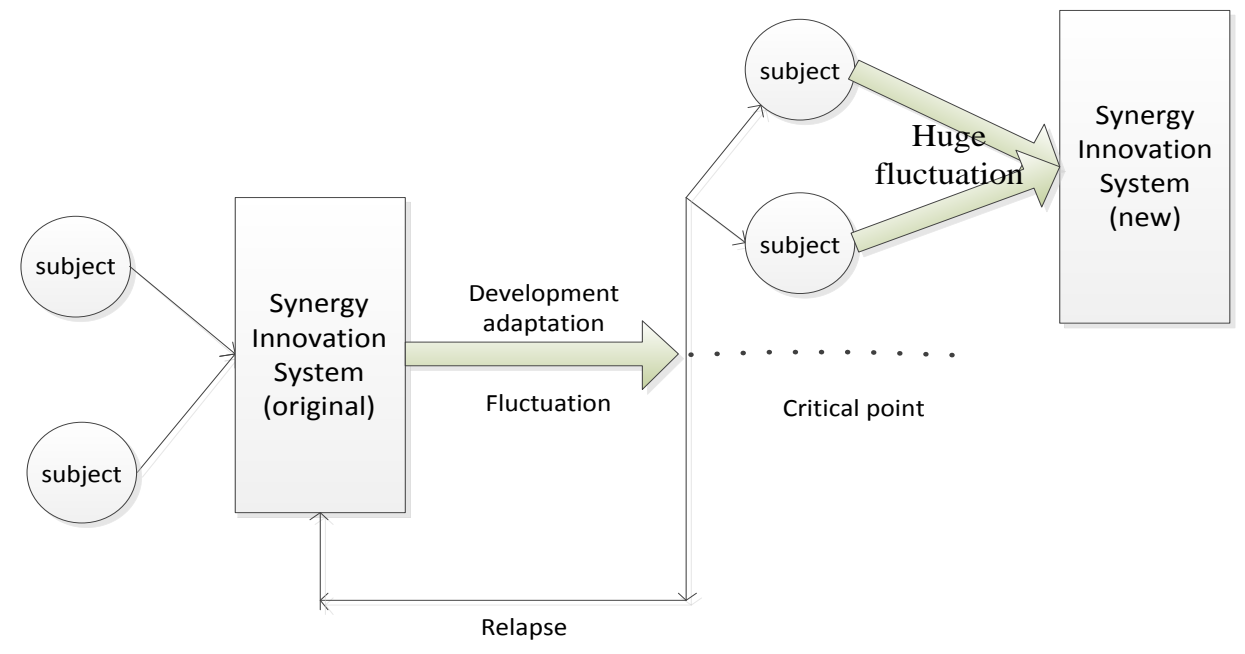

Fig.1 Self-evolution of Innovation System

\section{Synergy Model of the Urban Agglomeration Innovation}

Profit is the most simple and direct motive of any economic activity. Only when the synergy innovative income of the urban agglomeration is greater than zero, the innovation subject has the power to choose the synergy. This is easier to meet so the innovation subject is more inclined to synergy to create high profits. The effect of the synergy innovation of urban agglomeration obeys logistic law, the logistic equation can be used to establish the model of the synery innovation to analyze the synergy motive of innovation agents.

Assume that $\mathrm{P}$ standing for the effect of the synergy innovation and $\mathrm{M}$ standing for the extreme value of the effect of the independent innovation. The basic equation for Logistic is: $d P / d t=k P(1-P / M)$. dp/dt represents the growth rate of the effect of the synergy innovation, $\mathrm{k}$ is the growth factor. The synergy innovation of urban agglomeration is non-linear and there is positive and negative feedback mechanism, so $\mathrm{P} / \mathrm{M}$ is the correction factor. According to the requirement of actual economic meaning, there should have $\mathrm{M}>0, \mathrm{k}>0$.

Assuming two innovative subjects are $\mathrm{C} 1$ and $\mathrm{C} 2$. Respectively, $\mathrm{r} 1$ is the contribution rate of the innovation of $\mathrm{C} 1$ to $\mathrm{C} 2$, and $\mathrm{r} 2$ stands for $\mathrm{C} 2$ to $\mathrm{C} 1$. When $\mathrm{r} 1>0$ and $\mathrm{r} 2>0$, there will be

$$
\begin{aligned}
& d P_{1} / d t=k_{1} P_{1}\left(1-P_{1} / M_{1}+r_{2} P_{2} / M_{2}\right) \\
& d P_{2} / d t=k_{2} P_{2}\left(1-P_{2} / M_{2}+r_{1} P_{1} / M_{1}\right)
\end{aligned}
$$

When the synergy between the two sides is in equilibrium, the equation is zero. Solves the equation to obtain the equilibrium point $B_{1}(0,0), B_{2}\left(M_{1}, 0\right), B_{3}\left(0, M_{2}\right)$, 


$$
B_{4}\left(\frac{M_{1}\left(1+r_{2}\right)}{1-r_{1} r_{2}}, \frac{M_{2}\left(1+r_{1}\right)}{1-r_{1} r_{2}}\right)
$$

The equation is expanded by Taylor series, and the equilibrium point is brought into the coefficient matrix. The analysis shows that B1,B2 and $\mathrm{B} 3$ are not balance points, $\mathrm{B} 4$ is the balance point when $\mathrm{r} 1 * \mathrm{r} 2<1$. At this time, the innovation effect of $\mathrm{C} 1$ and $\mathrm{C} 2$ respectively exist in the following relationship:

$$
\frac{M_{1}\left(1+r_{2}\right)}{1-r_{1} r_{2}}>M_{1}, \frac{M_{2}\left(1+r_{1}\right)}{1-r_{1} r_{2}}>M_{2}
$$

Obviously, the extreme effect value of the innovation increases when both sides innovating to achieve a win-win situation. This model shows that the innovative advantages created by the synergy innovation encourage the innovation subject to synergy spontaneously to get more competitive.

\section{Self-Organizing Mechanism of the Synergy Innovation of the Urban Agglomeration}

There are many parameters affecting the change and development of the system. The order parameter derived from the internal system, describes the overall behavior of the system and plays a decisive role in the system. Innovation subjects or subsystem correlate in long range, forming partnership, leading to the appearance of the order parameter and become a force to dominate the system's evolution. System transformation or not is directly determined by the action degree of the control parameter on the system. Only when the control parameter reaches a critical value, the self-organization process is likely to appear in the system. The key to the synergy innovation of the urban agglomeration is building self-organization mechanisms of the synergy innovation under the influence of the order parameter and the control parameter. Positive feedback from self-organization could strongly promote organic combination and association of the innovative element of the urban agglomeration.

\section{Orderly Evolution}

For the synergy innovation system of the urban agglomeration, the system elements will all affect the evolution and development, in which the functional element guides and promotes the evolution. Therefore, a series of the resource, the technology, the talent and the capital and other functional elements, combined with the initial state and evolution demands of the innovative subject, all may become the order parameter.

Order parameters have the branch of fast variables and slow variables which collectively dominate the subject behavior, transforming from the imbalance to the balance and from the disorder to the order under the function of the "Fluctuation Enlargement". In different stages of the urban agglomeration evolution and different areas, different innovation subsystems play different roles, resulting in the interaction between the innovation subsystems through competing, eventually forming the only order parameter, which is a mode of the synergy innovation mode or innovation value-added leading the development and the orderly degree. Specifically, the interaction of innovation elements form an order parameter set which has one or more order parameters including the enterprise, the government, the university and research institution, the service organization, guiding the innovation system to a higher order state, as shown in Fig.2. 


\section{Control Evolution}

As for the synergy innovation system, the change of environment elements will affect the process of synergy innovation and even the results. The elements do not make the decisive influence, and they belong to the control parameter. As shown in Fig.3, differences between innovation subjects, innovation resources, element distribution and ownership, and the adaptability to the external environment and responsiveness all lead to inevitable competition and friction which are closely related with the gradient of industrial structure, resource distribution, element complementation and fluency, environment stability, policy system. Under the combined action of these elements, the internal energy of the system is weakened, and it begins to drive away from the equilibrium state. In order to maintain the equilibrium state, an internal mechanism and external forces are needed to make the urban agglomeration gain positive value. This is the synergy mechanism which comes from the mutual trust and continuous and effective communication and collaboration within the system, and the external force which are the innovation element flow, information flow, material flow etc.

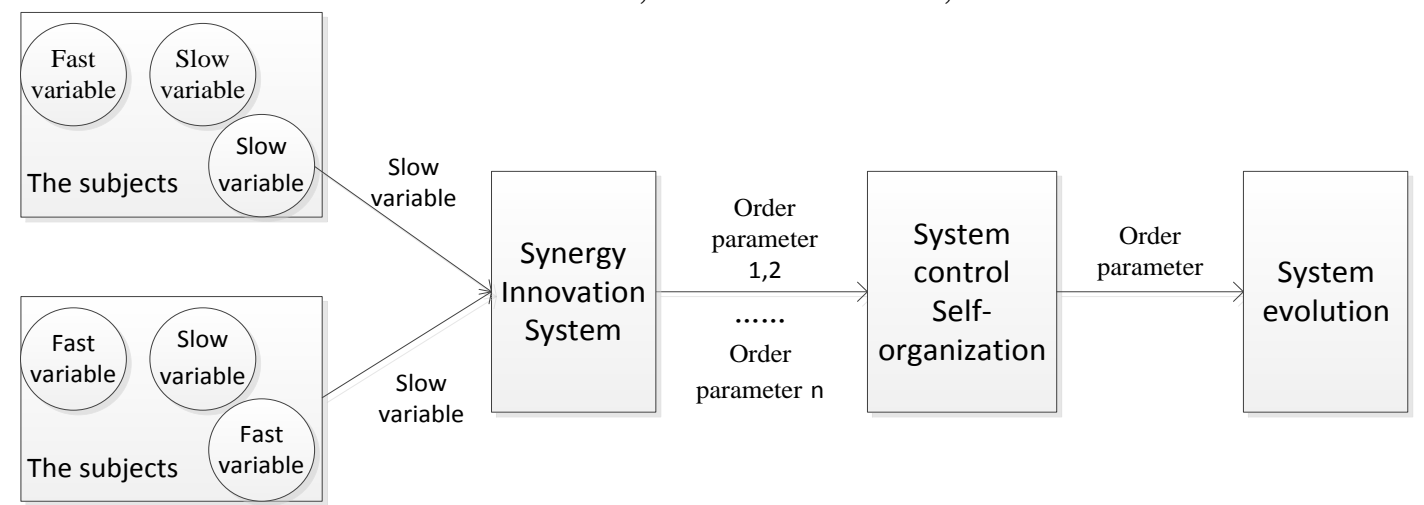

Fig.2 Orderly Evolution of Synergy Innovation System

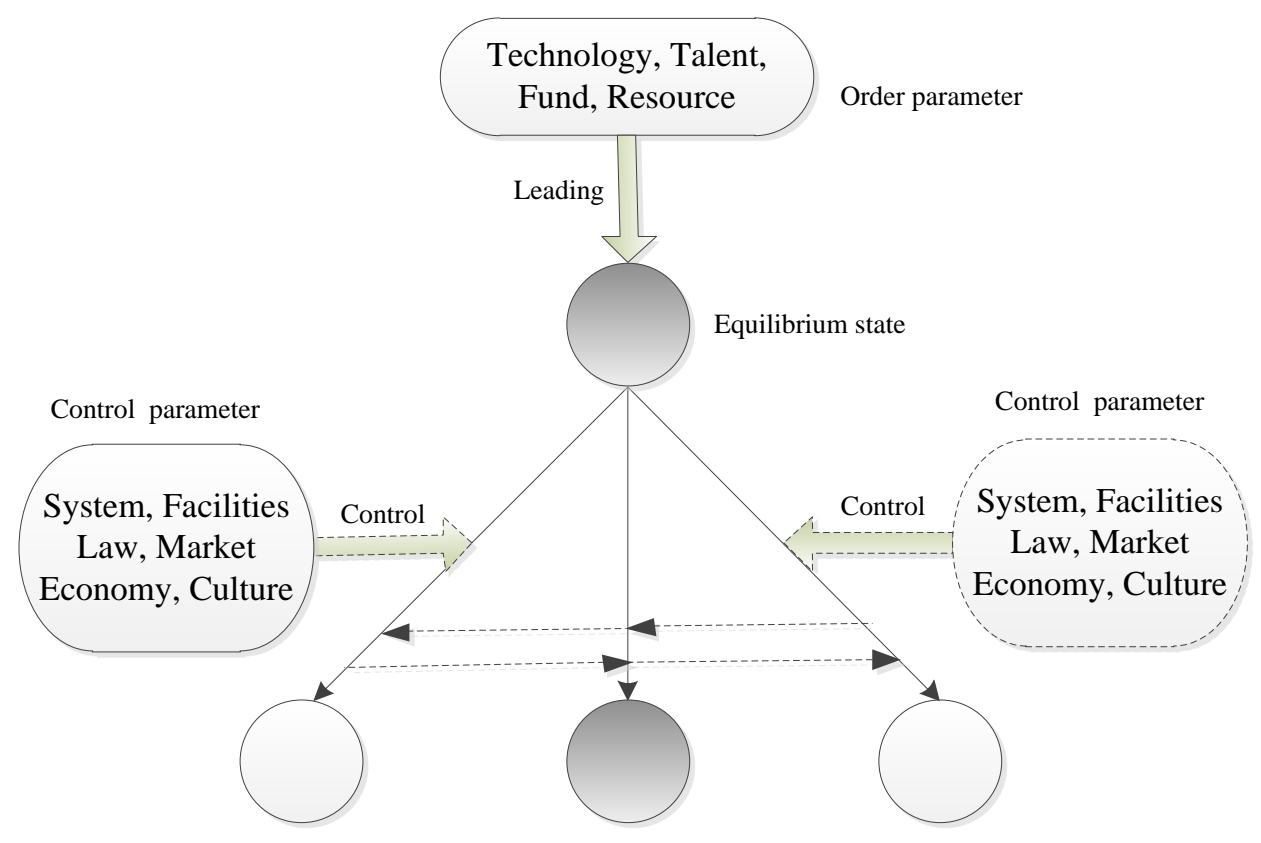

Fig.3 Control Evolution of Synergy Innovation System 


\section{Self-organization Mechanism}

The synergy innovation evolution of the urban agglomeration is a self-organization process. Since the formation of the structure of the self-organization is required to meet four conditions, openness, being far from equilibrium, nonlinear and fluctuation phenomena. Random fluctuation is the inducement of the self-organization evolution. In the process of the synergy innovation, there will be many microfluctuation factors such as innovation preference, creativity, market demand, science and technology development and so on, which would be amplified to form a macrofluctuation. The Nonlinear interaction is a key mechanism for the orderly evolution. Nonlinear effect makes elements in the system loss independence to form a the catalytic cycle of a two-way information transmission, so that the microfluctuation is more and more big until forming macrofluctuation. Open and non-equilibrium is a prerequisite, they fully open and exchange material, energy and information, obtain negative entropy flow such as capital, talent etc, far away from the equilibrium state in the role of macrofluctuation, provide transformation and evolution.

\section{Conclusion}

In this paper, the self-organization mechanism of the synergy innovation system of the urban agglomeration is analyzed. The components of the synergy innovation system can be divided into the subject element, the function element and the environment element, which are further described in this paper. On the basis of the basic theory of the Synergetics, characteristics of the innovation subject are analyzed including self-development, self-adaptation and self-evolution. The synergy model of the innovation subject is established, and the motivation and necessity of the synergy innovation are demonstrated, and the orderly evolution, the control evolution and the self-organization mechanism are analyzed. In this paper, the evolution of innovation subjects and the self-organization analysis are superficial. Next, the theory and demonstration analysis could be studied from the point of system phase transition and critical motion combining with the system dynamics and the game theory.

\section{Acknowledgement}

This research was financially supported by Humanities-Social Science Research project of Hubei Province Education Department (16G232) and Humanities-Social Science Research project of Ministry of Education (15YJC890015).

\section{References}

[1] Philip.Cooke, Regional innovation systems: competitive regulation in the new Europe, Geoforum. 3 (1992) 365-382.

[2] Veroniea.Serrano, Thomas.Fiseher, Collaborative Innovation in Ubiquitous Systems, International Manufacturing. 18 (2007) 599-615.

[3] Sheng Yuming, The impact of economic globalization and knowledge on global sustainable development, Economic Geography. 3 (1999) 13-17.(in Chinese)

[4] Xie Xuemei, Synergic innovation mechanism of metropolitan region:a regional innovation perspective based on synergism theory, Studies in Philosophy of Science and Technology. 1 (2011) 95-99. (in Chinese) 
[5] Ren Jianhua,Bai Sijun,Guo Yuntao, Research on the evolution and collaboration model of general aviation industry system in China on self-organization theory, Shanghai Management Science. 4 (2015) 51-55. (in Chinese)

[6] Cheng Qiang, Shi Linna, Study on mechanism of the industry-university-research institute synergetic evolution based on the self-organization theory, Soft Science. 4 (2016) 22-26. (in Chinese) 